\title{
A TURBULENT MIXTURE THEORY FOR THE ATMOSPHERIC MIXTURE OF SNOW AND AIR
}

by

\author{
Rand Decker and R. L. Brown \\ (Department of Civil Engineering and Engineering Mechanics, Montana State University, \\ Bozeman, Montana 59717, U.S.A.)
}

\section{ABSTRACT}

A theory based on classical fluid mechanics for an incompressible, chemically non-reacting, atmospheric mixture of air and entrained snow particles is derived. These continuum equations of motion are then expanded to include turbulent flow. The reduced, onedimensional equations of this theory are further refined by order-of-magnitude analysis and correlation of the turbulent terms to mean flow parameters. The resulting one-dimensional, turbulent equations of motion for the snow contain apparent turbulent forces which enhance entrainment of snow where gradients of the air flow are high. These turbulent equations of motion are then solved numerically for snow particle velocity and concentration as a function of height above the surface. The results are similar to observed profiles of snow concentration and the superposition of the solution of this turbulent inixture theory for snow entrainment with an appropriate solution for the saltation layer will eventually lead to a working continuum theory for blowing snow.

\section{THEORY}

The following continuum mixture theory for entrained snow in an atmospheric flow is based on the classical fluid mechanics principles of conservation of mass (continuity) and conservation of momentum for an incompressible, chemically non-reacting mixture (Hill and others 1980). Consider the flow of a mixture of $\mathrm{N}$ constituents. For three-dimensional mixture motion the equations of motion for the $\xi$ th constituent $(1<\xi<N)$ are:

$$
\dot{\phi}+\phi \nabla \cdot U_{\xi}=0 \text { (continuity) }
$$

and

$$
\rho_{\xi} \dot{U}_{\xi}=\rho_{\xi} \underline{\mathrm{b}}+\underline{\mathrm{D}}_{\xi}+\nabla \cdot{\underset{\sim}{\xi}}_{\boldsymbol{\xi}} \text { (momentum) , }
$$

where $T_{\xi}$ is the stress tensor for the $\xi$ th constituent, $\tilde{\rho}_{\xi}$ is mass density of the $\xi$ th constituent per unit volume of the mixture, $\phi=\rho \xi / \rho_{\text {ref } \xi}$ is the dimensionless concentration of the $\xi$ th constituent, Pref $\xi$ is a reference density indicative of the mass density per unit volume that the $\xi$ th constituent would achieve in a static condition, and $\phi$ is the substantive derivative of the concentration of the $\xi$ th constituent. $U_{\xi}$ is the velocity vector of the $\xi$ constituent, $\dot{U}_{\xi}$ is the substantive derivative of the velocity vector of the $\xi$ constituent, and b is body acceleration vector, which acts on all constituents. Finally, $D_{\xi}$ is the drag force of the $\xi$ th constituent, which is a vector-valued function of the concentration of the $\xi$ th constituent and the other constituents of the mixture.

For the case of an atmospheric mixture of snow particles and air in three-dimensional motion, the following set of twelve equations result:

$$
\begin{aligned}
& \dot{\phi}_{\mathrm{S}}+\phi_{\mathrm{S}} \underline{\nabla} \cdot \underline{U}_{\mathrm{S}}=0 \\
& \dot{\phi}_{\mathrm{a}}+\phi_{\mathrm{a}} \underline{\nabla} \cdot \underline{\mathrm{U}}_{\mathrm{a}}=0 \\
& \rho_{\mathrm{s}} \underline{\dot{U}}_{\mathrm{S}}=\rho_{\mathrm{S}} \underline{\mathrm{b}}+\underline{D}_{\mathrm{s}} \\
& \rho_{\mathrm{a}} \underline{\dot{U}}_{\mathrm{a}}=\rho_{\mathrm{a}} \underline{\mathrm{b}}+\underline{D}_{\mathrm{a}}+\nabla \cdot \stackrel{\mathrm{T}}{\mathrm{a}}_{\mathrm{a}} .
\end{aligned}
$$

The subscripts a and $s$ indicate the air constituent and the entrained snow, respectively.

The form and extent to which D participates as a force in the momentum equation is the result of constitutive assumption. For the case of an air/snow mixture, the following form is adopted:

$$
\underline{D}_{a}=-\underline{D}_{s}=\phi_{S} D\left(\underline{U}_{S}-\underline{U}_{a}\right),
$$

where $D$ is a numerical constant of ten referred to as a drag coefficient. In other words, the transfer of momentum between the air and the snow is a vector function of snow-particle concentration and the velocity differences between the air and the snow particles.

It would be possible to manipulate these equations for solutions for $U_{A}, U_{S}$ and $\phi_{S}$; however, they would lack the accuracy of a theory which takes into account the forces resulting from turbulent motion superimposed on these mean flows.

Adopting the standard notation for turbulent fluctuations of the mean flow parameters (Hinze 1959), we write:

$$
\begin{aligned}
& \underline{U}_{\mathrm{a}}=\underline{\mathrm{U}}_{\mathrm{a}}+\underline{U}_{\mathrm{a}}^{\prime} \\
& \underline{U}_{\mathrm{S}}=\bar{U}_{\mathrm{S}}+\underline{U}_{\mathrm{s}}^{\prime} \\
& \phi=\bar{\phi}+\phi^{\prime} \\
& \rho_{\mathrm{S}}=\bar{\rho}_{\mathrm{S}}+\rho_{\mathrm{S}}^{\prime},
\end{aligned}
$$


where, for example, $\bar{U}_{a}$ is the time-average value of the air-velocity vector, and $U_{a}^{\prime}$ is the turbulent fluctuation of the air velocity vector about its own time-average value.

Additional time averages can be taken of any product or sum of mean and fluctuating flow parameters; however, the following conventions (Hinze 1959) must be observed. If $f$ and $g$ are functions of time,

$$
\begin{aligned}
& \overline{\overline{f f}}=\bar{f} \\
& \bar{f} \pm \bar{g}=\overline{f \pm g} \\
& \overline{\overline{f g}}=\overline{f g} \\
& \overline{\frac{\partial f}{\partial x}}=\frac{\partial \bar{f}}{\partial x} \\
& \overline{f^{\prime}}=0 \\
& \overline{\left(f^{\prime} g^{\prime} f^{\prime}\right)}=0 \\
& \overline{f^{\prime} g^{\prime}} \neq \overline{f^{\prime}} \bar{g}=0 \\
& \overline{\left(f^{\prime} g^{\prime} f^{\prime} g^{\prime}\right)} \neq 0 .
\end{aligned}
$$

Substituting the functions of Equation (8) into the mean flow parameters in the continuity equation for the snow (Equation (3)) results in:

$$
\frac{\mathrm{d}}{\mathrm{dt}}\left(\bar{\phi}+\phi^{\prime}\right)+\left(\bar{\phi}+\phi^{\prime}\right) \underline{\nabla} \cdot\left(\underline{\mathrm{U}}_{s}+\underline{U}_{s}^{\prime}\right)=0 \text {. }
$$

Expanding the substantive derivative:

$$
\begin{gathered}
\frac{\partial}{\partial t}\left(\bar{\phi}+\phi^{\prime}\right)+\left(\underline{U}_{S}+\underline{U}_{S}^{\prime}\right) \cdot \underline{\nabla}\left(\bar{\phi}+\phi^{\prime}\right)+ \\
+\left(\bar{\phi}+\phi^{\prime}\right) \underline{\nabla} \cdot\left(\underline{U}_{S}+\underline{U}_{S}^{\prime}\right)=0 .
\end{gathered}
$$

Expanding and separating the terms into mean flow and turbulent fluctuation parameters:

$$
\begin{aligned}
& \frac{\partial \phi}{\partial t}+\underline{\mathbb{U}}_{S} \cdot \underline{\nabla \bar{\phi}}+\bar{\phi} \underline{\nabla} \cdot \underline{\bar{U}}_{S}+\frac{\partial \phi^{\prime}}{\partial t}+\underline{U}_{S}^{\prime} \cdot \underline{\nabla}^{\prime}+\phi^{\prime} \underline{\nabla} \cdot \underline{U}_{S}^{\prime}+ \\
& +\underline{\bar{U}}_{S} \cdot \underline{\bar{\nabla}} \phi^{\prime}+\underline{U}_{S}^{\prime} \cdot \underline{\nabla} \bar{\phi}+\bar{\phi} \underline{\nabla} \cdot \underline{U}_{S}^{\prime}+\bar{\phi}^{\prime} \underline{\nabla} \cdot \underline{\tilde{U}}_{S}=0 .
\end{aligned}
$$

The above may be rewritten in the form:

$$
\begin{aligned}
& \frac{\partial \bar{\phi}}{\phi t}+\underline{\nabla} \cdot\left(\bar{\phi} \underline{\tilde{U}}_{S}\right)+\frac{\partial \phi}{\phi t}+\underline{\nabla} \cdot\left(\phi^{\prime} U_{S}\right)+ \\
& +\underline{\nabla} \cdot\left(\bar{\phi} \underline{U}_{S}^{\prime}+\phi^{\prime} \underline{\widetilde{U}}_{S}\right)=0 .
\end{aligned}
$$

At this point it is advantageous to take the time average of each term in Equation (13), resulting in the third and fifth terms being identically zero as per the time average conventions:

$$
\frac{\partial \bar{\phi}}{\delta t}+\underline{\nabla} \cdot\left(\bar{\phi} \underline{\underline{U}}_{S}\right)+\underline{\nabla} \cdot\left(\bar{\phi}^{\top} \underline{\underline{U}}_{S}\right)=0 \text {. }
$$

Expanding the second terrn:

$$
\frac{\partial \bar{\phi}}{\partial t}+\underline{\tilde{U}}_{S} \cdot \underline{\nabla} \bar{\phi}+\bar{\phi} \underline{\nabla} \cdot \underline{U}_{S}+\underline{\nabla} \cdot\left(\bar{\phi}^{\prime} \underline{U}^{\top}\right)=0 .
$$

Rewriting the first and second terms as a substantive derivative:

$$
\frac{\bar{d} \bar{\phi}}{d t}+\bar{\phi} \underline{\nabla} \cdot \underline{U}_{s}+\underline{\nabla} \cdot\left(\bar{\phi}^{\prime} \underline{U}^{\top}\right)=0 .
$$

Note that the sum of the first and second terms is the continuity equation for the mean flow parameters and this sum has already been established (Equation (3)) as zero.

This leads to the identity:

$$
\underline{\nabla} \cdot\left(\bar{\phi}^{\top} \underline{U}_{S}^{\top}\right)=0 \text {, }
$$

and hence the turbulent continuity equation for the snow can be written solely as a function of mean flow parameters:

$$
\frac{\partial \bar{\phi}}{\partial t}+\underline{\mathbb{U}}_{S} \cdot \underline{\bar{\nabla}} \bar{\phi}+\bar{\phi} \underline{\bar{\nabla}} \cdot \underline{\bar{U}}_{S}=0
$$

or

$$
\frac{\partial \bar{\phi}}{\partial t}+\underline{\nabla} \cdot\left(\bar{\phi} \underline{U}_{S}\right)=0 .
$$

Substituting the mean flow and fluctuating turbulent parameters (Equation (8)) into the mean flow parameters of the monentum equation for the snow (Equation (5)) and the constitutive drag force (Equation (7)) and combining Equations (5) and (7) results
in:

$$
\begin{gathered}
\left(\bar{\rho}_{s}+\rho^{\prime}{ }_{s}\right)\left(\frac{d}{d t}\left(\underline{U}_{s}+\underline{U}_{s}^{\prime}\right)\right)=\left(\bar{\rho}_{s}+\rho_{s}^{\prime}\right) \underline{b}- \\
-\left(\bar{\phi}+\phi^{\prime}\right) D\left(\underline{U}_{s}+\underline{U}_{s}^{\prime}-\underline{\bar{U}}_{a}-\underline{U}_{a}^{\prime}\right) .
\end{gathered}
$$

Expanding the substantive derivative on the left side, expanding the distributive terms and noting that it will be advantageous to take an additional time average of all the resulting terms,

$$
\begin{aligned}
& \bar{\rho}_{s} \frac{\partial \underline{U}_{s}}{\partial t}+\bar{\rho}_{s} \frac{\partial \underline{\underline{U}}_{S}^{\prime}}{\partial t}+\rho_{s} \underline{\bar{U}}_{s} \cdot \underline{\nabla} \underline{\bar{U}}_{s}+\bar{\rho}_{s} \underline{\bar{U}}_{s} \cdot \underline{\nabla}_{\underline{U}_{S}^{\prime}}+
\end{aligned}
$$

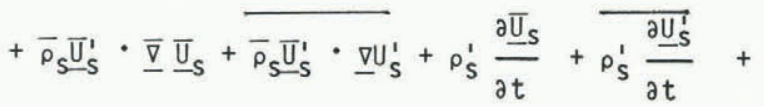

$+\bar{\rho}_{S} \underline{\bar{U}}_{S} \cdot \underline{\nabla} \underline{\bar{U}}_{S}+\overline{\rho_{S}^{\prime} \bar{U}_{S} \cdot \underline{\nabla} \underline{U}_{S}^{\prime}}+\overline{\rho_{S}^{\prime} \underline{U}_{S}^{\prime} \cdot \underline{\nabla}_{\bar{U}}}+$

$$
+\rho_{S}^{\prime} U_{S}^{\prime} \cdot \underline{\nabla} \underline{U}_{S}^{T}
$$

$=\overline{\left(\overline{\rho_{s}}+\rho_{s}^{\prime}\right)} \underline{b}-\bar{\phi} D\left(\underline{U}_{s}-\underline{U}_{a}\right)-\overline{\bar{\phi} D\left(\underline{U}_{s}^{\prime}-\underline{U}_{a}^{\prime}\right)}-$

$-\overline{\phi^{\prime} D\left(\underline{\bar{U}}_{s}-\underline{\bar{U}}_{a}\right)}-\overline{\phi^{\prime} D\left(\underline{U}_{s}^{\prime}-\underline{U}_{a}^{\prime}\right)}$.

By the time-average conventions, terms two, four, five, seven, and twelve on the left side are identically zero. Also, on the right side, time averages of fluctuations in terms one, three and four are identically zero. Therefore

$\bar{\rho}_{S} \frac{\partial \underline{\underline{U}}_{S}}{\partial t}+\bar{\rho}_{S} \underline{\tilde{U}}_{S} \cdot \underline{\bar{\nabla}} \underline{\tilde{U}}_{S}=\bar{\rho}_{S} \underline{b}-\bar{\phi} D\left(\underline{U}_{S}-\underline{\tilde{U}}_{\mathrm{a}}\right)-\underline{F}_{T}$,

where 
$\underline{F}_{T}=\overline{\rho_{S}^{\prime} \frac{\partial \underline{\underline{U}}_{S}^{\prime}}{\partial t}}+\underline{\underline{U}}_{S} \overline{\rho_{S}^{\prime} \cdot \underline{\nabla} \underline{U}_{S}^{\prime}}+\overline{\rho_{S}^{\prime} \underline{U}_{S}^{\prime}} \cdot \underline{\nabla}_{\underline{U}_{S}}+$

$+\bar{\rho}_{s}{\underline{U_{s}^{\prime}} \cdot \underline{\nabla}_{\underline{U}}^{\prime}}_{s}+\overline{\phi^{\prime} D\left(\underline{U}_{s}^{\prime}-\underline{U}_{a}^{\prime}\right)}$.

Equation (21) is the turbulent momentum equation for the snow. The terms in $\mathrm{F}_{T}$ all contain even ordered products of fluctuations. The apparent forces described by these terms are analogous to the apparent or Reynold's forces of the turbulent Navier-Stokes equations for fluid flow.

Equation (18), which is the turbulent continuity equation, and Equation (21), which is the turbulent inomentum equation, are the three-dimensional turbu1ent equations of motion for the snow.

The flow regime can be established by expanding the equations of motion for the air (Equations (4) and (6)) by turbulent analysis and solving simultaneously with the turbulent equations of motion for the snow (Equations (18) and (21)). The flow parameters $\phi$, I and $\mathrm{I}_{\mathrm{a}}$ can therefore be deterinined for all space variables.

As an initial step to testing turbulent mixture theory the turbulent equations of motion for the snow are reduced to the one-dimensional case. In onedimensional flow there are gradients of the flow parameters in only one direction. This is analogous to boundary-layer flow over an infinite flat surface with the body force $\rho_{\mathrm{s}}$. being parallel to the directions of the gradients.

In the following, using Cartesian coordinates, the positive $Y$ axis is vertical and the only body acceleration is $-b_{y}$. Gradients of flow parameters do not exist in the $X$ and $Z$ directions, and transient effects are neglected.

Applying the above constraints to the turbulent equations of motion for the snow (Equations (18) and (21)) results in

$$
\frac{d}{d y}\left(\bar{\phi} \bar{v}_{S}\right)=0
$$

and

$$
\bar{\rho}_{S} \bar{v}_{S} \frac{d}{d y} \bar{v}_{S}=-\bar{\rho}_{S} b_{y}-\bar{\phi} D \bar{v}_{S}-F_{T y} .
$$

It is possible to solve this $Y$ component of the turbulent momentum equation and the one-dimensional, turbulent continuity equation for the snow-fall velocity $v_{s}$ and the snow concentration $\phi$ as a function of height for turbulent mixture flow over an infinite flat surface.

First, however, it would be advantageous to examine the relative orders of magnitude of the terms in FTy and then write the remaining terms as functions of the mean flow parameters (Drew 1975).

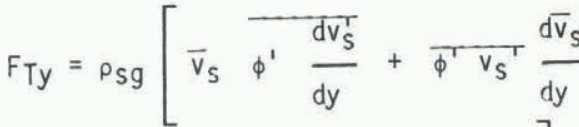

$$
\begin{aligned}
& \left.+\bar{\phi} v^{\prime} s \frac{d v_{s}^{r}}{d y}+\frac{D}{\rho_{s g}} \frac{\phi^{\prime} v_{s}^{r}}{\text {. }}\right] \text {, }
\end{aligned}
$$

where $\rho_{S g}$ is the mass density of deposited (static) snow. The relative orders of magnitude of the parameters in $\mathrm{F}_{\mathrm{Ty}}$ are

$$
\begin{aligned}
\overline{\mathbf{v}}_{\mathrm{S}} & \sim 1 \\
\bar{\phi} & \sim 1 \\
\mathbf{v}_{\mathrm{S}}{ }^{\prime} & \sim \delta \\
\phi^{\prime} & \sim \delta \\
\mathrm{y} & \sim \delta,
\end{aligned}
$$

where $\delta$ is a parameter much smaller than 1. Then, substituting these values for $F_{y}$,

$\mathrm{F}_{\mathrm{Ty}}=\rho_{\mathrm{Sg}}\left[\frac{1(\delta)}{(\delta)}+(\delta)(\delta) \frac{1}{\delta}+1(\delta) \frac{(\delta)}{(\delta)}+(\delta)(\delta)\right]$

Only the fourth term of $\mathrm{F}_{T y}$ is negligible relative to the other three terms.

The following constitutive assumptions are made for the values of the turbulent, fluctuating flow parameters in FTy as functions of the mean flow parameters. These constitutive assumptions follow from the arguments of Prandtl's mixing-length theory for correlation of turbulent, fluctuating flow parameters to mean flow parameters for homogeneous turbulence (Hinze 1959, Drew 1975).

$$
\begin{aligned}
& \phi^{\prime}=\bar{\phi} \varepsilon \frac{d \bar{U}_{a}}{d y} \\
& v^{\prime} s_{s}=\gamma \frac{d \bar{U}_{a}}{d y},
\end{aligned}
$$

where $\varepsilon$ and $\gamma$ are numeral constants with dimensions $t$ and $L$, respectively. In other words, these correlations state that the magnitude of any fluctuating parameter of the mixture can be no greater than the fluctuations of the fluid velocity. Substituting Equations (28) into Equation (25) (with the negligible terin dropped):

$F_{T y}=\rho_{s g}\left[\bar{v}_{s} \bar{\phi} \varepsilon \frac{d \bar{U}_{a}}{d y} \frac{d}{d y}\left(\gamma \frac{d \bar{U}_{a}}{d y}\right)+\right.$

$\left.+\bar{\phi} \gamma \frac{d \bar{U}_{a}}{d y} \gamma \frac{d \bar{U}_{a}}{d y} \frac{d \bar{v}_{s}}{d y}+\bar{\phi} \gamma \frac{d \bar{U}_{a}}{d y} \frac{d}{d y}\left(\gamma \frac{d \bar{U}_{a}}{d y}\right)\right]$.

Expanding the derivatives:

$\mathrm{F}_{T y}=\rho_{S y}\left[\varepsilon \gamma \bar{\phi} \quad \bar{v}_{S} \frac{d \bar{U}_{\mathrm{a}}}{d y} \frac{d^{2} \bar{U}_{a}}{d y^{2}}+\right.$

$\left.+\varepsilon \gamma \bar{\phi}\left(\frac{d \bar{U}_{a}}{d y}\right)^{2} \frac{d \bar{v}_{s}}{d y}+\gamma^{2} \bar{\phi} \quad \frac{d \bar{U}_{a}}{d y} \frac{d^{2} \bar{U}_{a}}{d y^{2}}\right]$.

Note that the apparent forces of turbulence in Equation (3) are particularly effective when the air velocity gradients and the particle velocity are strongest.

In summary, the one-dimensional, continuity equations and the equation of motion in the $y$ direction can be written:

$$
\frac{d}{d y}\left(\bar{\phi} \bar{v}_{s}\right)=0
$$

and

$$
\begin{aligned}
& \bar{\rho}_{S} \bar{v}_{S} \frac{d \bar{v}_{S}}{d y}=-\bar{\rho}_{S} b_{y}-\bar{\phi} D \bar{v}_{S}-\varepsilon \gamma \bar{\rho}_{S} \bar{v}_{S} \frac{d \bar{U}_{a}}{d y} \frac{d^{2} \bar{U}_{a}}{d y^{2}}- \\
& -\varepsilon \gamma \bar{\rho}_{S}\left(\frac{d \bar{U}_{a}}{d y}\right)^{2} \frac{d \bar{v}_{S}}{d y}-\gamma^{2} \rho_{S} \frac{d \bar{U}_{a}}{d y} \frac{d^{2} \bar{U}_{a}}{d y^{2}} .
\end{aligned}
$$


2. SOLUTION OF THE ONE-DIMENSIONAL TURBULENT EQUATIONS OF MOTION FOR THE SNOW

Examination of Equation (31), the turbulent continuity equation for the snow, leads to

$$
\phi \overline{\mathrm{V}}_{\mathrm{S}}=\text { constant. }
$$

This requires the snow-particle fall velocity to vary inversely with the particle concentration. Equation (32), the turbulent momentum equation for the snow, can be solved for $v_{s}$, the snow-particle fall velocity, as a function of height.

For an air velocity the established logarithmic profile was adopted (Geiger 1965, Plate 1971)

$$
\bar{U}_{\mathrm{a}}(y)=\frac{U_{*}}{\mathrm{k}} \operatorname{lN}\left(\frac{\mathrm{Y}_{\mathrm{Y}}}{\mathrm{Y}_{0}},\right.
$$

and it follows that

$$
\begin{aligned}
& \frac{d}{d y} \bar{U}_{a}(y)=\frac{U_{\star}}{k} \frac{1}{Y} \\
& \frac{d^{2} U_{a}}{d y^{2}}=-\frac{U_{\star}}{k} y-2,
\end{aligned}
$$

where $U_{*}, k$, and $Y_{0}$ are respectively the friction velocity, the von Karman constant, and the roughness height. Rewriting the turbulent momentum equation for the snow,

$\bar{v}_{s} \frac{d \bar{v}_{s}}{d y}+g+\frac{D}{\rho_{s g}} \bar{v}_{s}-\varepsilon \gamma\left(\frac{U_{\star}}{k}\right)^{2} \frac{1}{\gamma^{3}}+$

$+\varepsilon \gamma\left(\frac{\left.U_{*}\right)^{2}}{k}{ }_{\gamma^{2}} \frac{d \bar{v}_{s}}{d y}-\gamma^{2} \frac{U_{*}{ }^{2}}{k}\right)^{2} \frac{1}{\gamma^{3}}=0$.

This is a non-linear differential equation of the form

$$
\bar{v}_{S} \bar{v}_{S}^{\prime}+A(Y) \bar{v}_{S}^{\prime}+B \bar{v}_{S}=C(Y) \text {. }
$$

This equation was solved by finite difference techniques for $\bar{v}_{S}$, which represents the snow-fall velocity as a function of height over a vertical interval of $2 \mathrm{~m}$. The snow-fall velocity was calculated for an initial fall of $1.0 \mathrm{~m} \mathrm{~s}^{-1}$ for three different windspeed profiles. These results for $\bar{v}_{S}$ as a function of height were then fitted with the logarithmic function curves, summarized below:

(a) $\bar{U}_{a}(10 \mathrm{~m})=9.2 \mathrm{~m} \mathrm{~s}^{-1}, U_{\star}=0.8, \mathrm{k}=0.4, Y_{0}=0.1$, $\bar{v}_{S}(Y)=0.22 \log \frac{Y}{0.05}+0.63$

(b) $\bar{U}_{\mathrm{a}}(10 \mathrm{~m})=12.5 \mathrm{~m} \mathrm{~s}^{-1}, U_{\star}=0.5, \mathrm{k}=0.4$, $Y_{0}=0.0004, \bar{v}_{S}(Y)=3.11 \log \frac{Y}{0.05}+0.59$

(c) $\bar{U}_{\mathrm{a}}(10 \mathrm{~m})=16.0 \mathrm{~m} \mathrm{~s}^{-1}, U_{\star}=0.63, \mathrm{k}=0.4$, $Y_{0}=0.0004, \bar{v}_{S}(Y)=0.142 \log \frac{Y}{0.05}+0.53$.

The turbulent continuity equation (Equation (33)) can then be solved for the snow concentration profile using these known snow-fall velocity profiles.

A graphical plot of $\phi$ and attendant windspeed and fall velocity profiles is presented in Figure $1(a-c)$.

\section{CONCLUSIONS}

The solution to the turbulent one-dimensional equations of motion for an air-snow mixture are

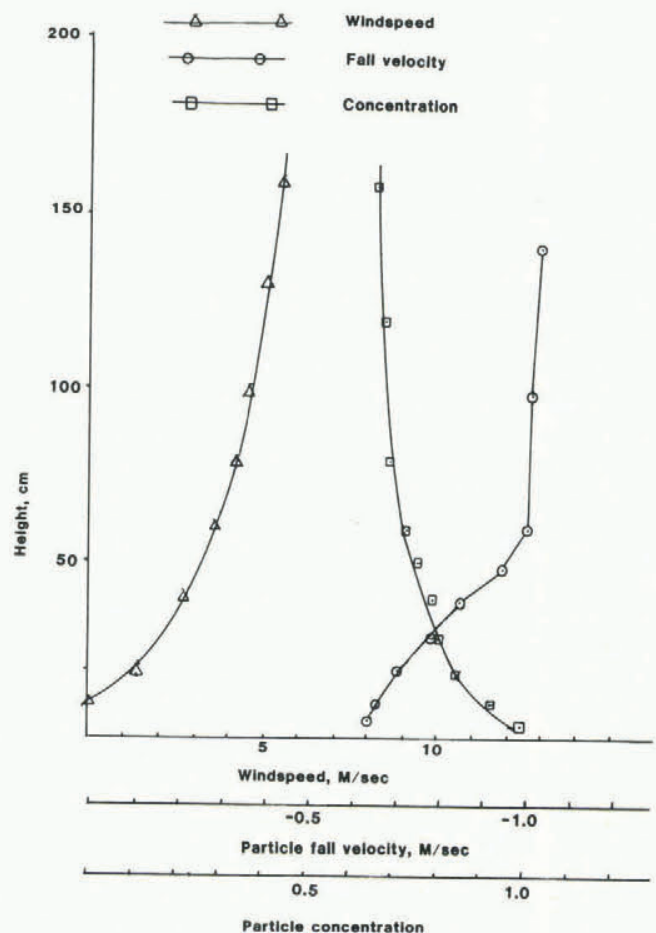

Fig.1(a). Horizontal windspeed, particle fall velocity and particle concentration vs height above the surface from the solution of the one-dimensional inixture theory equations. $U_{a 10}=9.2 \mathrm{~m} \mathrm{~s}^{-1}, U_{\star}=0.8$, $k=0.4, Y_{0}=0.1$.

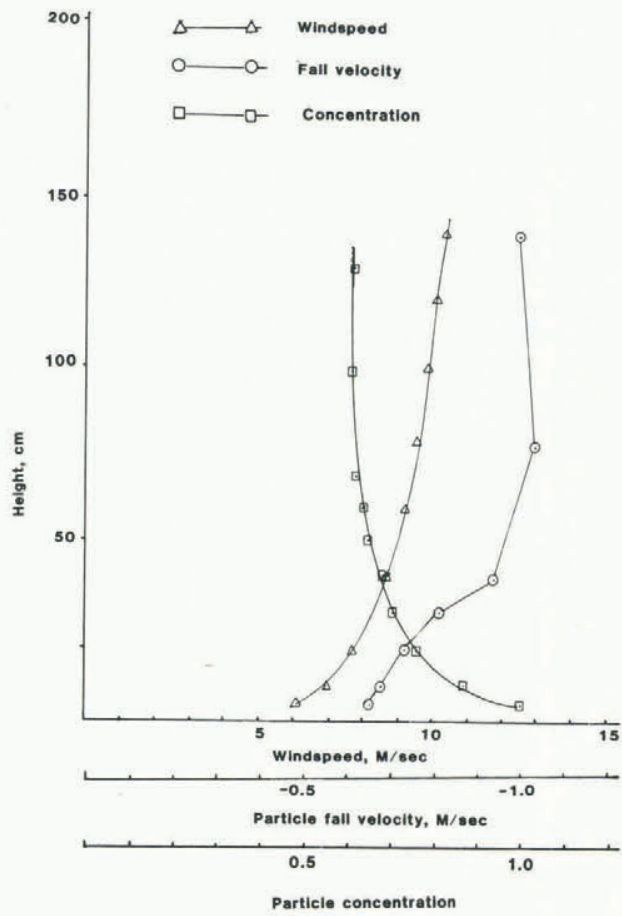

Fig.1(b). Horizontal windspeed, particle fall velocity and particle concentration vs height above the surface from the solution of the one-dimensional mixture theory equations. $U_{a 10}=12 \mathrm{~m} \mathrm{~s}^{-1}, U_{\star}=0.5$, $k=0.4, Y_{0}=0.0004$. 


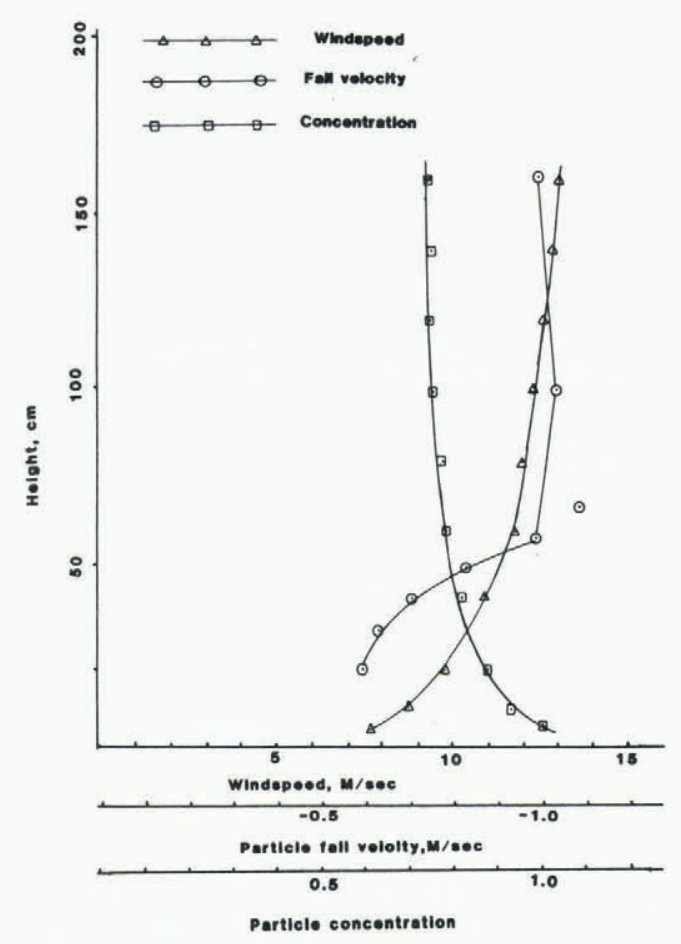

Fig.1(c). Horizontal windspeed, particle fall velocity and particle concentration vs height above the surface from the solution of the one-dimensional mixture theory equations. $U_{a 10}=16 \mathrm{~m} \mathrm{~s}^{-1}, U_{\star}=0.63$, $k=0.4, Y_{0}=0.0004$.

encouraging. The solution shows that turbulence due to strong air-flow gradients in the vicinity of a solid surface allows the snow-particle fall velocity to decrease and subsequently the entrained snow

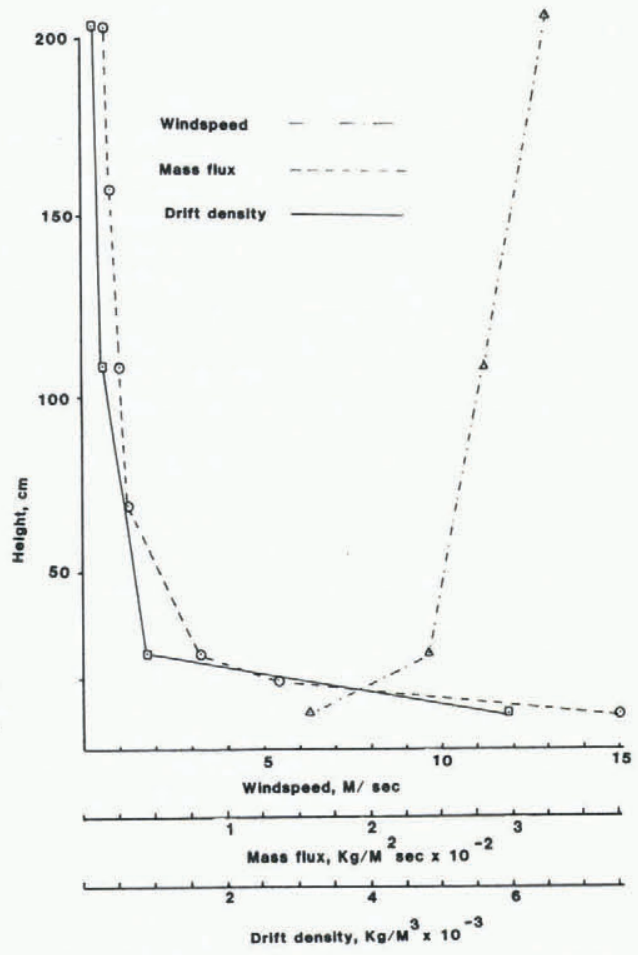

Fig.2(a). Windspeed, drifting snow mass flux and drift density vs height above the surface (Cuckoo's Nest research site, Bridger Bowl, Montana, 12 March 1982). $U_{1 \mathrm{~m}}=11.2 \mathrm{~m} \mathrm{~s}^{-1}$.

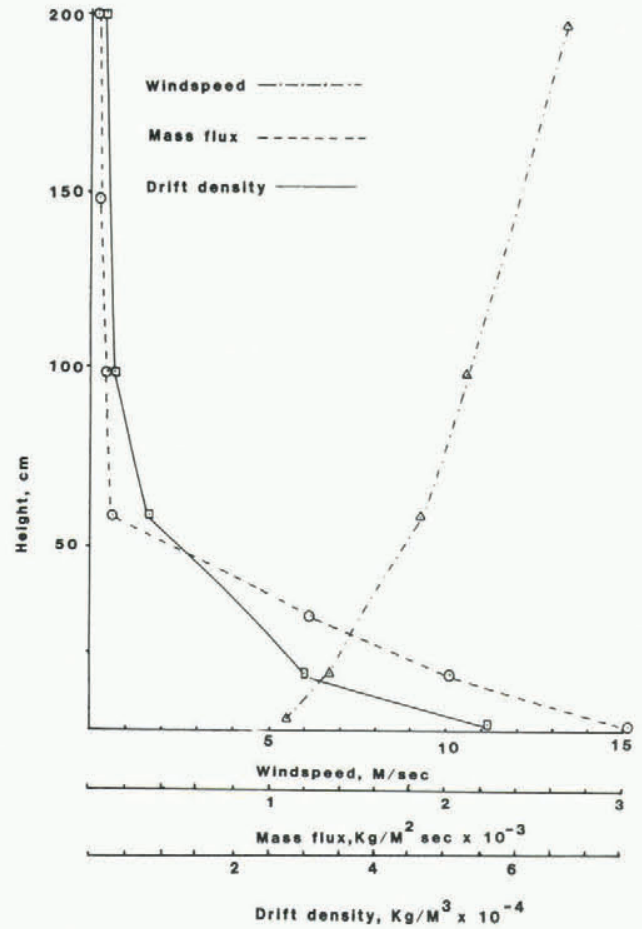

Fig.2(b). Windspeed, drifting snow mass flux and drift density vs height above the surface (Cuckoo's Nest research site, Bridger Bowl, Montana,12 March 1982). $\mathrm{U}_{1 \mathrm{~m}}=10.7 \mathrm{~m} \mathrm{~s}^{-1}$.

concentration to increase. These results are consistent with drifting snow mass flux and density profiles measured on the windward fetch of a mountain ridge in Montana (Fig.2(a) and (b)).

As the mechanics of the saltation layer 10 to 15 cm above surface) becomes better understood the superposition of this mixture theory solution for turbulent entrainment and transport with a solution for the saltation layer will lead to a general continuum theory for blowing snow.

In the case of two- and three-dimensional air/ snow turbulent mixture motion, there is an opportunity to determine wind-aided deposition or accumulation rates of new snow in stagnation regions of the flow such as on the lee of mountain slopes or in the lee of structures.

\section{ACKNOWLEOGMENTS}

The work reported here has been supported by US National Science Foundation (NSF) Grant No. ENG-7901636. The authors appreciate the continued support of the NSF.

\section{REFERENCES}

Drew D A 1975 Turbulent sediment transport over a flat bottom using momentum balance. Transactions of the ASME. Ser E. Journal of Applied Mechanics $42(1): 38-44$

Geiger R 1965 The climate near the ground. Cambridge, MA, Harvard University Press Hill C D, Bedford A, Drumheller D S 1980 An application of mixture theory to particulate sedimentation. Transactions of the ASME. Ser $E$. Journal of Applied Mechanics 47(2): 261-265

Hinze J O 1959 Turbulence. New York, McGraw-Hill Plate E J 1971 Aerodynamic characteristics of atmospheric boundary layers. AEC Critical Review Series 